\title{
La conceptualización del salario en las distintas vertientes de teoría económica
}

\author{
Gallo, Marcos Esteban* \\ *Universidad Nacional de Mar del Plata, Mar del Plata, Argentina \\ E-mail:marcosgll@yahoo.com.ar \\ https://orcid.org/0000-0002-3409-2076
}

Recibido: 7 de mayo de 2020

Aprobado: 15 de junio de 2020

\section{Resumen}

El presente trabajo procura llevar a cabo una reflexión teórica acerca de la conceptualización de los salarios reales y nominales en algunas de las principales doctrinas económicas. En tal sentido, se realiza un análisis comparativo de las diferentes concepciones sobre el salario, predominantes en la teoría neoclásica, la teoría keynesiana y el pensamiento marxista. Al respecto, para el pensamiento neoclásico los salarios se ven prioritariamente influidos por las fuerzas del mercado, mientras que para la teoría keynesiana guardan relación inversa con el nivel de empleo, de acuerdo a los principios de la productividad marginal del trabajo. Por su parte, para el pensamiento marxista resulta central la fase del ciclo en que se encuentre la economía, así como el estado de la lucha de clases. Asimismo, se estudia el modo en que determinados modelos de acumulación pueden imponer límites al intervalo dentro del cual pueden variar los salarios reales, haciendo referencia a algunas problemáticas específicas de la economía argentina, como la restricción externa de la economía y la orientación predominantemente exportadora y/o mercado internista de la estructura productiva.

Palabras clave: Salarios nominales, Salarios reales, Doctrinas económicas 


\title{
The conceptualization of wages in different economic theories
}

\begin{abstract}
This paper attempts to carry out a theoretical reflection on the conceptualization of real and nominal wages in some of the main economic doctrines. In this sense, a comparative analysis is made of the different conceptions on wages, which predominate in neoclassical theory, Keynesian theory and Marxist thought. In this regard, for neoclassical thinking, wages are primarily influenced by market forces, while for Keynesian theory they are inversely related to the level of employment, according to the principles of marginal productivity of labour. Instead, for Marxist thought, the phase of the economic cycle, as well as the state of the class struggle, are central. This paper also studies how certain accumulation models may impose limits on the range within which real wages may vary, referring to some specific problems of the Argentine economy, as the external constraint of the economy and the predominantly export orientation and/or domestic market orientation of the production structure.
\end{abstract}

Keywords: Nominal wages, Real wages, Economic doctrines.

\section{Introducción}

En la teoría económica existen dos cuestiones que son centrales en todo análisis: la capacidad del sistema para arribar $-\mathrm{o}$ al menos para tenderpor sí mismo al pleno empleo de los recursos sin intervención externa, y el rol que juegan los salarios en el proceso de ajuste. En ambos casos se trata de cuestiones con fuertes implicancias sociales. El primer punto remite a la discusión sobre la naturaleza del desempleo y el diagnóstico pertinente, con el consecuente impacto sobre el diseño de las políticas económicas que se consideran aplicables. El segundo se vincula de manera directa con el anterior, y remite al carácter del salario como factor instrumental de la política económica y como determinante de la distribución del ingreso.

Entre los múltiples criterios de clasificación que pueden establecerse, es posible dividir a las distintas vertientes teóricas en dos grandes grupos. Por un lado, aquellas que consideran al salario como una variable determinada endógenamente por el sistema, como una variable que tendría una magnitud técnicamente "correcta", en el sentido de que permitiría al sistema arribar a una posición de equilibrio estable con pleno empleo. En esta línea se inscribe la teoría neoclásica.

Por otro lado, existe un conjunto de corrientes teóricas que consideran al salario como un elemento determinado de manera exógena. En este sentido, se considera que el salario se establece de manera esencialmente institucional, entendiendo este concepto en el sentido amplio del término. Esto significa que lo institucional abarca tanto estructuras reglamentarias formales, como factores culturales y políticos en los que se refleja la relación de fuerzas entre distintos grupos de interés. Ello no implica negar la influencia que tienen sobre el salario las relaciones de oferta y demanda, pero sí supone negar la existencia de un nivel salarial óptimo que permita al sistema arribar a una situación de equilibrio armónico, con pleno empleo de los recursos y sustentable en el tiempo. Se concibe, en cambio, al desempleo involuntario como una posibilidad verosímil y perdurable. Las distintas vertientes heterodoxas de la teoría económica, como los modelos marxistas o neomarxistas, o las múltiples interpretaciones poskeynesianas comparten este criterio.

A medio camino entre ambas posturas consideramos que se encuentra la teoría keynesiana en su versión original, por las razones que se desarrollarán más adelante.

En el presente trabajo se procura realizar una reflexión teórica acera de la naturaleza que reviste 
el salario en las principales doctrinas económicas, incursionando esencialmente en el pensamiento de los autores básicos o fundacionales de cada una de ellas. Así, a partir de una revisión de la bibliografía existente sobre la materia, se hace hincapié en los postulados básicos referentes a la temática de la determinación salarial de la teoría económica neoclásica, el pensamiento keynesiano y la teoría marxista. Asimismo, se analiza también la manera en que distintos condicionantes estructurales históricamente determinados pueden incidir en la configuración de la estructura salarial, destacando fundamentalmente aquellos factores que han estado presentes en distintas etapas de la historia económica argentina.

\section{Planteamiento general del modelo neoclásico}

En el "mainstream" del pensamiento económico de las últimas décadas el modelo neoclásico se ha impuesto como la visión dominante ${ }^{1}$. En términos genéricos, este esquema teórico concibe al sistema económico como un conjunto de mercados interconectados debienesy de factores de producción, que puede arribar mediante mecanismos que le son intrínsecos a una situación de equilibrio general; esto es, una situación en la cual las cantidades de factores y de productos intercambiadas en el mercado, y sus correspondientes precios, quedan determinados de forma endógena y estable por el propio sistema, sin que operen fuerzas desestabilizadoras.

En el proceso que guía al sistema hacia el equilibrio general intervienen multitud de decisiones individuales adoptadas libremente según el criterio de la racionalidad, de modo que el equilibrio económico alcanzado en condiciones de libre decisión constituye un óptimo en el que las partes en cuestión obtienen el máximo beneficio posible ${ }^{2}$.
En términos metodológicos, el pasaje desde la órbita de las decisiones individuales a las determinaciones del sistema en su conjunto puede realizarse mediante la agregación de magnitudes individuales -lo que se conoce como individualismo metodológico-, sin que se plantee la posibilidad de contradicción entre la conveniencia individual y el bienestar general (Kicillof, 2007). Y gracias al accionar de mecanismos cuyo análisis se considerará más adelante en el presente apartado, el equilibrio general se corresponde con el pleno empleo de los recursos disponibles.

La explicación neoclásica del mercado laboral constituye una pieza más dentro del esquema de equilibrio general de inspiración walrasiana (Neffa, 2007). En términos analíticos, la forma de abordar el mercado de trabajo consiste en una particularización del mecanismo básico de interacción entre oferta y demanda que gobierna a todo el sistema. Se niega por lo tanto, la posibilidad de que el mercado laboral presente alguna característica específica que lo diferencie de los mercados de productos (Monza, 1981). La analogía con el resto de los mercados requiere de una función de demanda que relacione la cantidad demandada de factor trabajo con su precio, esto es, el salario real. Asimismo, se necesita una función de oferta en la cual la cantidad ofertada de horas de trabajo se vincule funcionalmente con el salario real.

La construcción de la función de demanda se deriva del concepto técnico de productividad marginal del trabajo, es decir, el incremento en el producto debido a un aumento unitario en la cantidad de factor trabajo empleado. La productividad marginal del trabajo se considera sujeta a la ley de rendimientos marginales decrecientes, de modo que, dado un stock fijo de capital plenamente utilizado, incrementos sucesivos en la cantidad de

1. Las ideas esenciales de la teoría económica neoclásica fueron sentadas por Alfred Marshall en su obra Principles of Economics. An introductory volume (1890). En lo referido a la teoría neoclásica del mercado de trabajo, constituye una obra emblemática el libro de Arthur Pigou Theory of Unemployment (1933). Al decir de Keynes (1974, p. 248) en relación a la obra de Pigou: “[...] el suyo es el único intento que conozco de desarrollar la teoría clásica de la ocupación en forma precisa. Así, me he visto obligado a dirigir mis objeciones contra esta teoría en la exposición más formidable que de ella se ha hecho.

REVISTA DE INVESTIGACIÓN SIGMA / Vol. 07, No 1, 2020 (pág. 6-21) 
factor trabajo empleado darán lugar a incrementos progresivamente menores en el volumen de producción. En este esquema los empresarios contratarán más trabajadores -o emplearán más horas-hombre de trabajo- siempre que el producto adicional generado por la última unidad de trabajo empleada sea superior a la remuneración percibida por esa última unidad de trabajo. Sólo de esa manera los capitalistas incrementarían su masa de ganancias a medida que contratan mano de obra, y se estarían comportando de acuerdo al principio de racionalidad.

Como la productividad del trabajo está sujeta a la ley de rendimientos marginales decrecientes, con cada unidad adicional de factor trabajo que se contrate el salario real deberá descender de manera que se iguale al producto marginal del trabajo para ese volumen dado de ocupación. Cuanto más bajo sea el salario real, mayor será la cantidad de factor trabajo que a los empresarios les resultará rentable emplear. De esta manera queda establecida una relación funcional en la que la cantidad de trabajo demandada se relaciona inversamente con el salario real. Así, a un menor salario real corresponde un mayor nivel de empleo, dado un determinado stock de capital que se utiliza plenamente -o sea, no hay capacidad ociosa-, y manteniendo constante el estado de la técnica. Los cambios tecnológicos o los incrementos en la demanda de trabajo debidos a la acumulación de capital pueden ser representados mediante cambios paramétricos en la función que se traducen gráficamente en desplazamientos de la curva de demanda de trabajo. Pero lo que ésta última busca remarcar, en definitiva, es la relación inversa entre la magnitud de los salarios reales y el nivel de empleo. Asimismo, los cambios técnicos que incrementan la productividad del trabajo -lo que se refleja gráficamente en un desplazamiento hacia arriba de la función de productividad marginal del trabajo- constituyen la única alternativa consistente para lograr un aumento de los salarios reales sin introducir interferencias distorsivas en los mecanismos del mercado.

En cuanto a la función de oferta de trabajo, está formulada de manera tal que los trabajadores buscan igualar el salario real con la desutilidad marginal que les ocasiona cada hora de trabajo adicional. De manera axiomática se postula que el sacrificio que implica el trabajo, formalizado en el concepto de desutilidad, crece proporcionalmente más que el tiempo que se destina al trabajo, de modo que la desutilidad marginal del trabajo se incrementa a medida que aumenta la cantidad de factor trabajo empleado. Para compensar esa desutilidad marginal creciente se debe pagar un salario real mayor a medida que se emplea un volumen más grande de factor trabajo. Así, la función de oferta de trabajo relaciona positivamente la cantidad de trabajo ofertada con el nivel del salario real. Aquellos cambios estructurales que pueden influir en la oferta de trabajo, como el crecimiento vegetativo de la población, transformaciones demográficas de magnitud como las debidas a movimientos migratorios o conflictos armados, o cambios culturales que pueden incidir

2. A lo largo del siglo XX se han elaborado múltiples replanteos de la teoría neoclásica, entre los que cabe destacar a la síntesis neoclásica, el monetarismo y la escuela de las expectativas racionales. La exposición más acabada de la síntesis neoclásica puede hallarse en el célebre libro de Don Patinkin (1959) Dinero, interés y precios. Los postulados esenciales de la escuela monetarista pueden consultarse en distintos textos de la vasta obra de Milton Friedman, entre los cuales pueden destacarse Studies in the Quantity Theory of Money (1956), A Program for Monetary Stability (1960), The Optimun Quantity of Money and Other Essays (1969), o el más conocido A Monetary History of the United States, 1867-1960 (1963), entre otros. La escuela de las expectativas racionales fue desarrollada principalmente a partir de los trabajos de Robert Lucas, Thomas Sargent y Neil Wallace, entre los que pueden mencionarse "Rational" Expectations, the Optimal Monetary Instrument, and the Optimal Money Supply Rule (Sargent y Wallace, 1975), After Keynesian Macroeconomics (Lucas y Sargent, 1979) y Rational Expectations and Econometric Practice (Lucas y Sargent, 1981). Todas estas escuelas comparten, en líneas generales, la confianza en el ajuste automático de los mercados y responsabilizan a la rigidez de precios y salarios por la persistencia de situaciones de desempleo involuntario. La escuela de las expectativas racionales directamente plantea la absoluta ineficacia de cualquier política pública. 
en el posicionamiento de determinados grupos de personas en relación al mercado laboral son representados mediante cambios paramétricos en la función que se reflejan gráficamente en el desplazamiento de la curva de oferta de trabajo.

A partir de las funciones de oferta de trabajo individuales se puede derivar la función de oferta de trabajo agregada sumando la totalidad de las horas de trabajo ofertadas por todos los individuos de la sociedad para cada nivel de salario real dado. De manera análoga, la suma de la totalidad de las horas de trabajo demandadas por todos los empresarios individualmente considerados para cada magnitud del salario real da lugar a la función de demanda de trabajo agregada. A partir de allí resulta fácil deducir el salario real de equilibrio del mercado de acuerdo a los típicos principios de interacción entre oferta y demanda. En el equilibrio el salario real iguala simultáneamente a la productividad marginal del trabajo y a la desutilidad marginal del mismo. Todos los agentes del mercado se encuentran en una situación óptima porque al salario real de equilibrio los empresarios no tienen incentivos para incrementar la cantidad de trabajo demandada ni los trabajadores encuentran motivos para aumentar la cantidad de trabajo ofertada.

Por eso mismo, el volumen de ocupación que se alcanza al nivel del salario real de equilibrio corresponde al pleno empleo de la fuerza de trabajo, porque todo el que esté dispuesto a aceptar el salario que se deriva de su contribución marginal al producto conseguirá emplearse, y sólo permanecerán desempleados quienes voluntariamente decidan permanecer fuera del mercado laboral debido a su disconformidad con el salario real que se les ofrece. Aquí resulta importante señalar que, dada la posición de las curvas de oferta y demanda de trabajo, el salario real funciona como un determinante causal del nivel de empleo; es decir, la relación causal va del salario real al volumen de empleo, y no al revés. Por esa razón, en el esquema neoclásico el único motivo posible por el que puede haber desempleo involuntario es la persistencia de un salario real superior al de equilibrio, determinado generalmente por factores de orden institucional exógenos al modelo, considerados como distorsionantes de las fuerzas naturales del mercado, que limitan la cantidad de factor trabajo que los empresarios están dispuestos a demandar. Por ende, la mejor política económica para promover el crecimiento del empleo resulta ser la eliminación de todos los elementos que impiden que el salario real se ubique libremente en su nivel de equilibrio.

Si se trasciende el punto de vista parcial que implica el análisis del mercado de trabajo tomado en forma aislada, se concluye que el salario real determinado según el libre juego de la oferta y la demanda constituye en realidad sólo un punto en el vector de precios que corresponde al equilibrio general del sistema en su conjunto, esto es, el equilibrio simultáneo de todos los mercados. Como, de acuerdo a los principios walrasianos que inspiran el concepto neoclásico de equilibrio general, una distorsión en un mercado afecta necesariamente a los demás mercados interrelacionados que componen el sistema, permitir que el salario real arribe a su nivel de equilibrio no es sólo una condición necesaria para que el mercado laboral logre el pleno empleo de la fuerza de trabajo, sino que también es un requisito indispensable para eliminar las distorsiones de precios y cantidades que se presenten en cualquier parte del sistema económico. Así, el salario real de equilibrio determinado según los principios neoclásicos constituye el nivel técnicamente "correcto"-el único nivel técnicamente "correcto"que permite alcanzar el óptimo social que implica el equilibrio general del sistema, y cualquier elemento que se interponga en el camino del ajuste

3. Por desutilidad debe entenderse cualquier motivo que induzca a un hombre o a un grupo de hombres a abstenerse de trabajar antes que aceptar un salario que represente para ellos una utilidad inferior a cierto límite" (Keynes, 1974, p. 17).

4. En la terminología original de la Teoría General Keynes denomina "escuela clásica" a lo que hoy se designa comúnmente como escuela neoclásica.

REVISTA DE INVESTIGACIÓN SIGMA / Vol. 07, No 1, 2020 (pág. 6-21)

ISSN 2631-2603 / Departamento de Ciencias Económicas, Administrativas y del Comercio

Universidad de las Fuerzas Armadas ESPE 
hacia el equilibrio debe ser eliminado, o por lo menos minimizado, a fin de maximizar el bienestar colectivo.

\section{La alternativa keynesiana frente a la ortodoxia}

\subsection{La crítica de Keynes al esquema neoclásico}

El principal libro de Keynes, la Teoría general de la ocupación, el interés y el dinero, comienza cuestionando el modelo neoclásico del mercado de trabajo, que era una de las facetas donde más expuesta quedaba la incapacidad de la economía ortodoxa para dar cuenta de la realidad imperante en el contexto de la Gran Depresión de los años `30, así como para proponer políticas económicas eficaces que permitieran al menos morigerar los efectos de la crisis (Kicillof, 2010; Pérez y Neffa, 2006).

Keynes resume este modelo -que él llama teoría clásica de la ocupación- en dos postulados fundamentales:

1. El salario es igual al producto marginal del trabajo.

2. La utilidad del salario, cuando se usa determinado volumen de trabajo, es igual a la desutilidad $^{3}$ marginal de ese mismo volumen de ocupación.

El primer postulado remite a la forma que en se construye la función de demanda de trabajo, mientras que el segundo hace referencia a la forma de derivar la función de oferta. Como se expuso en el apartado anterior, la interacción entre ambas funciones determina el salario real de equilibrio y el volumen de ocupación.
Keynes acepta el primer postulado, pero cuestiona el segundo, al que dirige una serie de críticas. Fundamentalmente, Keynes afirma que son dos las objeciones contra el segundo postulado de la escuela neoclásica ${ }^{4}$. En primer lugar, no se corresponde con los hechos que ante una reducción del salario real debida a un aumento de los precios se produzca una reducción en la cantidad de mano de obra ofertada. En segundo lugar, los obreros en su conjunto no tienen control sobre el salario real que perciben; sólo pueden establecer convenios sobre los salarios nominales, siendo los salarios reales una variable que depende de muchos otros factores.

En consecuencia, queda invalidada así la prescripción neoclásica que propone un recorte en los salarios reales como remedio necesario y suficiente para eliminar el desempleo involuntario, dejando que el mercado -Ley de Say mediantehaga todo lo demás 5 .

\section{2. Lineamientos principales de la Teoría General}

En respuesta a la evidente falta de correspondencia entre la teoría neoclásica y la realidad económica perceptible durante la Gran Depresión, Keynes propone un sistema teórico alternativo que parte de cuestionar la idea de que la oferta crea su propia demanda para cualquier nivel de ocupación.

El núcleo central de la teoría propuesta por Keynes se basa en admitir la posibilidad de que, por diferentes motivos, la comunidad en su conjunto no gaste la totalidad del ingreso. Como observa Keynes, cuando el nivel de ocupación aumenta, también lo hace el ingreso global de la comunidad. Sin embargo, debido a razones psicológicas, cuando

5. La Ley de Say afirma que en todo proceso productivo la suma de las remuneraciones a los factores es igual al valor del producto final, de modo que en el propio proceso de fabricación de un producto se crea el poder adquisitivo necesario para que ese producto sea comprado. Esto se resume comúnmente en el aserto que sostiene que la oferta crea su propia demanda. Si los empresarios tienen la certeza de que cada unidad adicional de producto que fabriquen podrá ser vendida, el producto y el empleo se expandirán sin necesidad de una intervención activa por parte del Estado, hasta que se alcance la plena utilización de los factores productivos. Por lo tanto, según la economía neoclásica, no es posible la existencia de situaciones duraderas de desempleo involuntario, a menos que los mecanismos de ajuste del mercado sean indebidamente interferidos. 
el ingreso se incrementa, el consumo crece en menor proporción. De este modo, a medida que aumenta el empleo, se genera una brecha creciente entre el consumo y el ingreso, que requiere ser compensada con un mayor volumen de inversiones. Sólo así podrá sostenerse el incremento de la ocupación (Keynes, 1974).

En consecuencia, la recomendación de Keynes para reducir el desempleo consiste en incentivar la inversión. Por su parte, la teoría de la inversión de Keynes se vincula con su teoría del dinero, la cual afirma que existen distintos motivos que pueden inducir a las personas a atesorar dinero.

Los tres motivos centrales mencionados por Keynes son transaccional, precaucional, y especulativo, prestando especial atención al tercero. En efecto, al plantear la posibilidad de una demanda especulativa de dinero, Keynes afirma que este último puede ser, además de medio de cambio, una reserva de valor, es decir, una de las formas que adopta el ahorro. En consecuencia, el atesoramiento de saldos líquidos pasa a tener un sentido en sí mismo y constituye una pérdida del sistema por la cual se fuga parte de la capacidad de compra que se genera al retribuir a los factores en el proceso productivo. Para Keynes, la demanda especulativa de dinero se encuentra inversamente relacionada con la tasa de interés, en tanto que esta última constituye una compensación por desprenderse del dinero a cambio de activos de menor liquidez.

Frente a la demanda especulativa de dinero, un incremento de la oferta monetaria por decisión del Banco Central provocará un exceso en las tenencias de saldos líquidos por parte de los particulares, parte del cual se canalizará hacia la compra de bonos, elevando así su precio y bajando, por lo tanto, la tasa de interés, hasta que las tenencias de dinero del público estén en equilibrio con el nuevo nivel de oferta monetaria ${ }^{6}$. El dinero, por lo tanto no es neutral, sino que puede tener un efecto concreto sobre el ingreso real y sobre el nivel de empleo mediante la incidencia que la tasa de interés ejerce sobre el volumen de inversiones.

El nivel de inversiones, a su vez, estará determinado por la interacción entre la tasa de interés y la eficiencia marginal del capital. Esta última se define como la tasa de descuento a la cual los rendimientos futuros estimados de la inversión igualan al monto de la inversión inicial. Aquí, nuevamente Keynes introduce un punto de ruptura con la economía neoclásica. Mientras que para esta última el capital tiene un ingreso cierto y calculable -su producto marginal- sobre el cual se estima la demanda de fondos prestables, para Keynes nada hay que asegure el éxito de una inversión. El rendimiento que en el futuro puede reportar un proyecto de inversión que se inicia hoy es apenas una esperanza sujeta a una infinidad de contingencias. Esta relación intertemporal entre la inversión y su ganancia, y el componente de incertidumbre que media entre ambos, hace que el monto global de inversiones pueda sufrir fuertes oscilaciones sin que haya causas objetivas aparentes para ello. Así, en el esquema keynesiano, la tasa de interés como compensación por la iliquidez, el dinero como posible reserva de valor y la incertidumbre con respecto al futuro condicionando el monto global de inversiones se unen para configurar la posibilidad del desempleo involuntario y persistente.

\section{3. Salarios reales y salarios nominales en la Teoría General}

La fundamentación de Keynes en contra de la tendencia del mercado a arribar por sí solo al pleno empleo conlleva una refutación del mecanismo neoclásico de determinación de salarios y empleo. En cambio, según este autor, una política eficaz de empleo basada en una flexibilidad a la baja de los salarios nominales debería estar necesariamente mediada por los efectos que estos últimos puedan tener sobre la propensión a consumir, sobre la eficiencia marginal del capital y/o sobre la tasa de interés.

6. Demandar bonos equivale a ofrecer préstamos, con lo cual un exceso de demanda de bonos se corresponde con un exceso de oferta de créditos, cuyo efecto será un descenso de la tasa de interés.

REVISTA DE INVESTIGACIÓN SIGMA / Vol. 07, No 1, 2020 (pág. 6-21)

ISSN 2631-2603 / Departamento de Ciencias Económicas, Administrativas y del Comercio

Universidad de las Fuerzas Armadas ESPE 
Respecto de la incidencia de una rebaja en los salarios nominales sobre la propensión a consumir Keynes es decididamente escéptico. En tal sentido, sostiene que los efectos regresivos en materia distributiva que tendría una política este tipo resentiría la propensión al consumo antes que incentivarla, con lo cual se agravarían los problemas de empleo.

En cuanto a las posibles consecuencias sobre la eficiencia marginal del capital, las mismas dependerán de las expectativas sobre la evolución futura de los salarios que tengan los empresarios. $\mathrm{Si}$ éstos esperaran que los salarios nominales suban en el futuro, se verían favorecidas las inversiones en el presente, dado que existiría la conjetura de que los costos serán mayores en el futuro. Lo contrario sucede si los inversores suponen que los salarios monetarios caerán aún más; las decisiones de inversión se verían aplazadas debido a que se creería que los costos actuales son superiores a lo que serán en el futuro. Además, en el caso de la fabricación de artículos para asalariados, menores salarios implican menores expectativas de ventas, con lo cual caería aún más la eficiencia marginal del capital. En cualquier caso, el efecto de un recorte de los salarios nominales sobre las inversiones es ambiguo y está sujeto a los débiles y cambiantes fundamentos sobre los cuales los empresarios basan sus expectativas.

Latasa de interésnominal puedeverse influenciada por una disminución de los salarios nominales en la medida en que ésta tenga consecuencias deflacionarias. En efecto, la reducción de precios derivada de una caída en los salarios monetarios incrementará la oferta real de dinero, y con ello se posibilitará una baja en la tasa de interés nominal que puede favorecer la inversión ${ }^{7}$. Sin embargo, una caída en los precios también incrementará el valor real de las deudas y la tasa de interés real, dificultando el pago de los créditos y pudiendo comprometer la solvencia de los deudores. Esto podría llevar a la quiebra a numerosas empresas y, en última instancia, aumentar aún más el desempleo. Por otra parte, en lo que a la tasa de interés nominal respecta, un recorte de los salarios monetarios equivale a un aumento en la oferta nominal de dinero, siendo infinitamente más fácil de implementar lo segundo que lo primero, dadas las dificultades de orden político y social que conllevaría una estrategia de rebaja generalizada de salarios. Por esta razón Keynes se opone a aquellos que confían en el ajuste automático de los mercados a partir de este mecanismo, y en lugar de ello aboga por una intervención activa del Estado a través de una política monetaria expansiva.

Finalmente, Keynes admite que, en una economía abierta, una rebaja de los salarios nominales locales en relación a los salarios nominales del exterior puede favorecer un crecimiento del empleo al alentar un saldo superavitario de la balanza comercial - estimulando así la inversión-, siempre y cuando esto implique una reducción de los costos de producción nacionales con respecto a los extranjeros. Sin embargo, dado que esto implica un abaratamiento relativo de los productos que un país exporta en relación a los artículos que importa, esta política también puede empeorar los términos del intercambio, lo que repercute en una reducción de

7. La caída en la tasa de interés nominal ocasionada por un recorte en los salarios monetarios es conocida en la literatura como "efecto Keynes". Algunas vertientes de la escuela poskeynesiana niegan que este fenómeno pueda tener lugar, ya que consideran que la oferta monetaria es endógena y se ajusta al nivel de la demanda de dinero mediante la dinámica del sistema crediticio. En lugar de controlar la oferta de dinero, el Banco Central determina exógenamente la tasa de interés nominal de corto plazo como instrumento de política monetaria (Moore, 1988; citado en Panigo, 2006). En estas condiciones el efecto Keynes no opera, porque ante la caída de los precios originada por el recorte de los salarios monetarios, en lugar de aumentar la oferta real de dinero disminuye la demanda nominal de dinero transaccional, contrayendo con ello la oferta monetaria nominal y dejando inalterada la tasa de interés nominal definida exógenamente por el Banco Central. Más aún, en estas condiciones aumentarían la tasa de interés real y el valor real de las deudas, con lo cual la disminución de los salarios nominales, lejos de incentivar la inversión y el empleo, operarían exactamente en el sentido contrario. 
los ingresos reales.

Teniendo en cuenta los argumentos precedentes, Keynes se manifiesta decididamente en contra de la posibilidad de favorecer el crecimiento del empleo mediante una reducción de los salarios nominales. Además de que tal estrategia sería impracticable en una sociedad democrática, de intentarse daría lugar a una gran inestabilidad de precios que haría difícil efectuar las previsiones requeridas por una economía de mercado. Por ello la recomendación de Keynes respecto de la política salarial es tratar de mantener los salarios nominales tan estables como sea posible (Keynes, 1974). Esta recomendación es además coherente con la posibilidad de los sindicatos, que sólo tienen capacidad para negociar con sus empleadores un determinado nivel de salarios nominales, siendo poco lo que pueden hacer para asegurarse un monto estable de salarios reales. En suma, en el esquema keynesiano, los salarios nominales requieren de una determinación institucional exógena (Kicillof, 2004), no siendo el ajuste salarial un mecanismo válido para incrementar el nivel de ocupación ${ }^{8}$.

Sin embargo, al aceptar el primer postulado de la teoría clásica de la ocupación, Keynes admite el precepto neoclásico según el cual el salario es igual a la productividad marginal del trabajo. De esta manera, aunque los salarios nominales permanezcan fijos en virtud de un arreglo institucional, el nivel de salarios reales se ajustará siempre para igualar a la productividad marginal del trabajo. En este esquema, si aumenta el nivel de ocupación, dado un stock de capital fijo, los precios subirán como un reflejo de la productividad marginal decreciente del trabajo, de modo que con salarios nominales estables, los salarios reales caerán conforme desciende la productividad del trabajo. Sin embargo, para Keynes, menores salarios reales son una consecuencia del incremento del empleo, pero nunca pueden ser su causa.

No obstante, la aceptación del primer postulado de la escuela clásica tiene una implicancia sobre la que es menester llamar la atención. Para afirmar que los salarios reales bajan cuando aumenta el empleo, el stock de capital utilizado debe permanecer invariable. De este modo, el concepto de desempleo referido en la Teoría General es unidimensional, ya que remite únicamente a desempleo de la fuerza de trabajo. El stock de capital, o bien es plenamente utilizado durante la depresión, o bien no se incorporan a la producción las unidades de capital ociosas a medida que aumenta el empleo. Cualquiera de las dos alternativas es completamente inverosímil. En cambio, si se admite que el nivel de utilización de capacidad instalada cae en la depresión y aumenta en la recuperación, acompañando los movimientos del nivel de empleo, no hay razón para argumentar que los salarios reales deben caer cuando se reduce la desocupación.

En un artículo publicado con posterioridad a la Teoría General Keynes reconoce esta objeción y afirma que el nivel de los salarios reales no es un determinante importante en las fluctuaciones cíclicas de corto plazo, a menos que se esté en una situación próxima al pleno empleo (Keynes, 1939; citado en Astarita, 2008). Aunque esta idea no invalida en términos lógicos el primer postulado de la teoría clásica de la ocupación, sí le quita relevancia práctica. De esta manera, no sólo el recorte salarial no es una política adecuada para promover el empleo, sino que tampoco es de esperar que la recuperación económica vaya acompañada de una caída de los salarios reales, con lo cual, de ningún modo la rigidez de los salarios a la baja puede ser señalada como el factor responsable de la persistencia de desempleo involuntario.

\section{Salarios, ganancias $y$ desempleo en Marx: la dinámica cíclica del capitalismo}

En la teoría marxista el análisis de los salarios, las ganancias y el desempleo aparece íntimamente ligado al concepto de la teoría del valor y de la dinámica cíclica de la economía capitalista. Si bien

8. En otro nivel de análisis, Canitrot (1983) coincide con la determinación esencialmente institucional de los salarios nominales. 
no se niega que las relaciones de oferta y demanda de empleo incidan en la dinámica de los salarios reales, éstos están determinados en primera instancia por el valor de cambio de la fuerza de trabajo.

Este concepto debe ser entendido en el marco de la ley general de valor, según la cual el valor de cambio de una mercancía está dado por el tiempo de trabajo socialmente necesario para producirla. Según Marx, "Es sólo la cantidad de trabajo socialmente necesario, pues, o el tiempo de trabajo socialmente necesario para la producción de un valor de uso, lo que determina su magnitud de valor" (Marx, 2004, p. 48, cursivas en el original). Aquí, el trabajo socialmente necesario se refiere al tiempo de trabajo promedio requerido para producir un valor de uso en las condiciones técnicas normales vigentes en una sociedad y con el nivel promedio de destreza e intensidad de trabajo.

Siguiendo este esquema, el valor de cambio de una jornada de fuerza de trabajo ${ }^{9}$ estará determinado también por el tiempo de trabajo socialmente necesario para producirla. El valor de la fuerza de trabajo está dado, a su vez, por su costo de reproducción, o sea, el valor de los bienes y servicios que típicamente consumen un trabajador y su familia durante una jornada completa. En palabras de Marx (2004, p. 207): "[...] el valor de la fuerza de trabajo es el valor de los medios de subsistencia necesarios para la conservación del poseedor de aquélla.” Aquí, el poseedor de la fuerza de trabajo es el trabajador, que la ofrece a cambio de los medios de subsistencia necesarios para él y su familia. Sin embargo, es importante remarcar que para Marx, el conjunto de los medios de subsistencia no se limita a las mercancías imprescindibles para la supervivencia biológica del trabajador y su familia, sino que la misma es una canasta de bienes y servicios determinada cultural e históricamente, y puede exceder en una medida importante el umbral de la supervivencia biológica (Starosta y Caligaris, 2017). El valor de cambio de esta cesta de bienes y servicios constituye el valor de cambio de la fuerza de trabajo, mientras que el valor de uso de esta última consiste en su capacidad de crear nuevo valor de cambio, es decir, valor de cambio expandido o plusvalor.

Sin embargo, la existencia de un valor de cambio determinado para la fuerza de trabajo que puede ser más o menos estable, especialmente en períodos cortos, no implica que el salario se establezca en una magnitud equivalente a dicho valor. En lugar de ello, el costo de reproducción de la fuerza de trabajo constituye un centro gravitatorio en torno del cual el salario real puede experimentar fuertes fluctuaciones que estarán condicionadas por la evolución del ciclo económico y por la relación de fuerzas entre el proletariado y los capitalistas propietarios de los medios de producción ${ }^{10}$. Asimismo, nada hay en el modelo planteado por Marx, que remita a alguna noción de pleno empleo como situación hacia la cual tienda el mercado de trabajo. Por el contrario, el desempleo de una parte significativa de la fuerza de trabajo es considerado como una situación normal, dando lugar a un ejército industrial de reserva o superpoblación relativa, cuya funcionalidad es central en la contención del nivel de salarios y en el mantenimiento de los márgenes de beneficio (González, 2011; Nun, 1969). De esta manera, tanto las fluctuaciones salariales como las variaciones coyunturales del nivel de empleo giran en torno de los cambios en el ejército industrial de reserva, dando lugar a un proceso fluctuante gobernado en última instancia por el ritmo y la forma que toma la acumulación de capital, y en el que se descarta toda

9. Es decir, el trabajo considerado en su forma dinámica, o sea, la disposición de un individuo a trabajar durante un tiempo determinado bajo las órdenes de otra persona que le provee la materia prima y los medios de producción necesarios.

10. En rigor, ambos factores están interrelacionados. En las fases ascendentes del ciclo económico el poder de negociación de los trabajadores se incrementa como consecuencia de la caída del desempleo, lo que posibilita la intensificación de la lucha por mejoras salariales. Lo contrario sucede en las fases descendentes del ciclo, en las que el aumento del desempleo erosiona la resistencia obrera a los recortes salariales, dando lugar a un incremento en la tasa de ganancia. De esta manera, las fluctuaciones del salario real en las distintas fases del ciclo económico se encuentran mediadas por las formas que asume la lucha de clases. 
idea de equilibrio estable.

Como explica Sweezy (1969), en el proceso normal de reproducción ampliada del capital tiene lugar una acumulación gradual de medios de producción que conlleva, en términos generales, un incremento en la demanda de fuerza de trabajo. Cuando la acumulación sobrepasa cierto límite, la superpoblación relativa tiende a agotarse y el poder de negociación de los trabajadores se incrementa, con lo cual la divergencia entre los salarios pagados y el valor de la fuerza de trabajo se amplía a favor de los obreros, afectando de manera adversa la tasa de ganancia del capital. Esta relación inversa entre salarios y ganancias capitalistas regula el ritmo de acumulación de capital, de manera tal que cuando la tasa de ganancia cae por debajo de determinado punto el proceso de acumulación se ralentiza o se detiene, expulsando mano de obra y permitiendo una recomposición del ejército industrial de reserva que elimina la presión al alza de los salarios ${ }^{11}$.

En abierta oposición a las teorías neoclásica y keynesiana, los salarios reales se mueven en forma procíclica, creciendo cuando el desempleo disminuye y viceversa, de manera tal que no se puede establecer una relación causal unidireccional entre salarios y desempleo en ninguno de los dos sentidos. Este punto es de crucial importancia, porque aquí el esquema marxista difiere de las otras teorías mencionadas, no sólo por las relaciones de causalidad que plantea, sino por su propia lógica de pensamiento. Así, mientras que para la teoría neoclásica el nivel del salario real es una causa determinante del nivel de empleo, para la teoría keynesiana éste último está gobernado por el nivel de demanda efectiva y el salario real es una variable dependiente determinada a su vez por el volumen de ocupación, invirtiendo la relación de causalidad neoclásica. Para Marx, en cambio, entre salarios y desempleo existe una relación dialéctica, en la cual el incremento del empleo genera, mediante el aumento procíclico del salario real, los desencadenantes de su propia negación. De la misma manera, al aumentar el desempleo los salarios reales caen, posibilitando las condiciones para que el empleo vuelva a subir. El volumen de empleo y el salario real son a la vez causa y efecto; ambos se condicionan mutuamente en un proceso cíclico mediado por la tasa de ganancia y por el ritmo de acumulación de capital, y donde el ejército industrial de reserva es la variable de ajuste del sistema.

Sin embargo, si bien el costo de reproducción de la fuerza de trabajo opera como centro gravitatorio de las variaciones del salario, ello no implica que quede determinado endógenamente en un nivel más o menos rígido. Por el contrario, el hecho de que el costo de reproducción de la fuerza de trabajo esté influenciado por cuestiones históricas y culturales, además de por razones técnicas referidas a los procesos productivos, le otorga un margen de flexibilidad que puede ser objeto de negociación entre trabajadores y capitalistas. En consecuencia, entre un mínimo de supervivencia biológica y un máximo que erosiona la tasa de ganancia al punto de imposibilitar la acumulación ampliada del capital, existe un amplio margen de negociación dentro del cual es posible mejorar la distribución del ingreso, lo cual dependerá fundamentalmente de la relación de fuerzas entre trabajadores y empresarios (Astarita, 2008; González, 2011). Así, la dinámica del ciclo económico en el sistema capitalista se encuentra mediada por el estado de la lucha de clases.

11. Según Serrano, la explicación marxista acerca de los mecanismos de recomposición del ejército industrial de reserva es coincidente con el análisis poskeynesiano sobre la determinación del nivel de empleo. "Para esta corriente de pensamiento [la teoría poskeynesiana] la obtención de beneficio también es la base de la inversión capitalista, componente fundamental de la demanda agregada. Si no existen expectativas de beneficio, los empresarios no invertirán o, en términos marxistas, no se iniciará el ciclo del capital avanzando dinero. En el corto plazo, la inversión depende de la estabilidad de determinados parámetros a largo plazo. Si el estado de la técnica es compatible con la obtención del beneficio deseado, el circuito del capital se iniciará con volumen suficiente, en caso contrario nos encontraremos en presencia de desempleo. [...] El conflicto por el reparto de la renta es lo que contribuye de manera más directa a la alteración de las expectativas de beneficio y, por esta vía, a las crisis económicas." (Serrano, 2006: 27).

REVISTA DE INVESTIGACIÓN SIGMA / Vol. 07, No 1, 2020 (pág. 6-21) 


\section{Determinación de los salarios y régimen de acumulación}

A fin de ponderar el alcance explicativo de cada perspectiva teórica, resulta ineludible considerar las características distintivas del régimen de acumulación cuyo estudio se aborda. En este sentido, el concepto de régimen de acumulación debe ser entendido como la "articulación de un determinado funcionamiento de las variables económicas, vinculando una definida estructura económica, una peculiar forma de Estado y las luchas entre los bloques sociales existentes" (Basualdo, 2007, p. 6). En efecto, cada formación económico-social en un determinado momento histórico presenta características estructurales específicas que obligan a matizar o reinterpretar el alcance explicativo de los distintos marcos teóricos.

En lo que respecta a la economía argentina, en diferentes períodos históricos su desarrollo se ha visto condicionado en mayor o menor medida por el problema conocido como restricción externa. En consonancia con esta problemática, algunos modelos que buscan explicar el comportamiento de la economía argentina han puesto en primer plano a esta problemática. En su forma básica (Braun y Joy, 1968; Canitrot, 1975 y 1983), el modelo de la restricción externa -también llamado modelo stop and $g o$ - fue concebido para dar cuenta de los factores que condicionaban el funcionamiento de la economía argentina durante el modelo de industrialización por sustitución de importaciones (ISI). Este régimen de acumulación se caracterizaba por el desarrollo de una estructura industrial orientada al abastecimiento del mercado interno y altamente dependiente de la provisión de insumos y capital importados, mientras que la producción exportable consistía esencialmente en productos agropecuarios, que son al mismo tiempo componentes fundamentales de la canasta básica de consumo de los asalariados, razón por la cual su precio es un determinante de primer orden del nivel de salario real. El modelo stop and go destaca que el incremento de los salarios reales presiona al alza los niveles de consumo, tanto de bienes industriales como, en menor medida, de productos agropecuarios, provocando un ascenso de las importaciones industriales y una disminución de los saldos exportables. Si la situación persiste, el alza de los salarios reales torna deficitaria la balanza comercial, provocando una pérdida de reservas internacionales. La recomposición del nivel de reservas obliga a implementar una política de estabilización cuyo efecto sobre el salario real es un recorte que lo sitúa en una magnitud compatible con un superávit de balanza comercial.

En definitiva, lo que el modelo destaca es la existencia de un límite superior para los salarios determinado por la necesidad de mantener el equilibrio de las cuentas externas (Fernández y González, 2019; Canitrot, 1983). Con una estructura industrial altamente dependiente de insumos y capital importados -como lo era la industria radicada en Argentina durante la fase desarrollista de la ISI en los años sesenta- ese límite puede estar por debajo del que determina una tasa de ganancia mínima que asegure la acumulación ampliada de capital, por lo menos en lo que respecta a las fracciones del capital dominantes. Asimismo, el límite máximo de los salarios impuesto por la restricción externa podría ser vulnerado, por lo menos temporalmente, en la medida en que exista acceso al financiamiento externo. En ese caso el techo salarial podría ser desplazado hacia arriba en tanto y en cuanto exista la posibilidad de conseguir refinanciamiento y siempre que la deuda externa no alcance una dinámica autopropulsada donde las nuevas líneas de crédito sólo alcancen para pagar los servicios de la deuda anterior. No obstante, un corte en las líneas de crédito exterior una vez que existe deuda externa acumulada agravaría la restricción externa, imponiendo un techo salarial aún más bajo que el que existiría en condiciones de ausencia de endeudamiento externo, porque entonces las exportaciones deberían financiar los servicios de la deuda acumulada con anterioridad, además de las importaciones necesarias para el funcionamiento de la estructura productiva.

En forma similar al incremento en los servicios de la deuda externa incidiría una fuga de capitales exacerbada, así como un flujo excesivo de remisión de utilidades al exterior por parte de empresas que operan en el país. En efecto, ambos fenómenos generarían una pérdida de divisas que -de no mediar

Gallo, Marcos Esteban 
la posibilidad de endeudarse con el exterior- debería ser compensada con resultados superavitarios de la balanza comercial, para lo cual resulta funcional una contracción salarial que comprima el nivel de consumo de los asalariados.

Sin embargo, como destacan Cortés y Marshall (2003), los modelos de stop and go en su versión tradicional tienen un sesgo en el sentido de que enfatizan en exceso el papel del consumo de los asalariados en la demanda de importaciones. En cambio, si se tiene en cuenta la influencia que ejerce el consumo de los sectores no asalariados de altos ingresos sobre las importaciones, puede concluirse que, hasta cierto punto, un incremento de los salarios podría aliviar la restricción externa en lugar de agravarla, dado que ello podría redistribuir ingresos desde sectores altamente demandantes de importaciones -los no asalariados de altos ingresoshacia otros que demandan proporcionalmente menos bienes del exterior -los asalariados-. Asimismo, tener en cuenta el rol que cumple el consumo de los sectores de altos ingresos abre otras posibilidades que obligan a matizar las conclusiones del modelo stop and go tradicional. En efecto, en la medida en que las clases propietarias destinen los ingresos provenientes de los beneficios y las rentas al gasto en consumo suntuario, el límite que la restricción externa impone al salario real se ve empujado hacia abajo por dos razones: en primer lugar porque se agrega un componente adicional a la demanda de importaciones que afecta negativamente el saldo de la balanza comercial; y en segundo lugar, porque se ve resentida la acumulación de capital que permitiría aliviar la restricción externa en el largo plazo mediante incrementos en la productividad.

Los factores considerados hasta aquí han hecho referencia a la existencia de un límite superior para los salarios reales. Dependiendo de las características de un determinado régimen de acumulación, el salario real podrá tener un techo impuesto por una tasa de ganancia mínima necesaria para asegurar cierto ritmo de expansión del capital, tal como afirma la teoría marxista, o podrá verse constreñido por las limitaciones que imponga la restricción externa de la economía en sus distintas variantes. De la misma manera, los salarios reales tienen un piso que dependerá de características particulares del aparato productivo y de la estructura demográfica que presente una formación económico-social, así como de la capacidad de negociación y de movilización política y organizativa de los asalariados, todo ello enmarcado en un régimen de acumulación específico (Amico, 2015). En tal sentido, uno de los factores que resultan cruciales para establecer el límite inferior hacia el cual pueden moverse los salarios reales estriba en cuáles son las fuentes de demanda a las que se enfrenta la estructura productiva. En este aspecto, una economía en la cual el consumo interno de los asalariados ocupe un lugar importante en la formación de la demanda efectiva tendrá restricciones para reducir los salarios reales más allá de determinado punto por debajo del cual el sistema entraría en recesión. En cambio, si la fuente central de demanda efectiva se ubica en el mercado externo, el salario real pasa a ser principalmente un costo de producción cuyo límite inferior sería el costo de reproducción de la fuerza de trabajo, entendida en el sentido marxista. Más aún, si una formación económico-social primordialmente exportadora posee además una amplia superpoblación relativa excedente, ya ni siquiera la reproducción de la fuerza de trabajo pasa a ser una prioridad para el capital, razón por la cual los salarios reales pueden directamente carecer de piso, o bien tener un piso determinado por la conflictividad social que tal situación generaría.

\section{Conclusiones}

Los diferentes marcos conceptuales analizados en el presente trabajo ofrecen distintas perspectivas a partir de las cuales pueden elaborarse explicaciones tentativas acerca de los procesos que subyacen en la formación de los salarios reales y nominales. Dichas perspectivas a veces confluyen al señalar a determinados factores como determinantes de los salarios, mientras que en algunas oportunidades aparecen divergencias profundas que tornan incompatibles los distintos puntos de vista.

En lo que respecta a la determinación de los salarios nominales, puede decirse que la misma depende en gran medida de factores institucionales y administrativos. Esto significa que en los mecanismos de formación de los salarios nominales resultan

REVISTA DE INVESTIGACIÓN SIGMA / Vol. 07, No 1, 2020 (pág. 6-21)

ISSN 2631-2603 / Departamento de Ciencias Económicas, Administrativas y del Comercio

Universidad de las Fuerzas Armadas ESPE 
centrales los marcos regulatorios establecidos por alguna autoridad estatal. Ello resulta claro cuando el monto de los salarios es definido directamente por alguna disposición reglamentaria del Estado o cuando predomina la negociación colectiva de convenios paritarios llevada a cabo conforme a una legislación específica. Pero incluso en el caso de la negociación descentralizada, ya sea por empresa o por trabajador, en la que la posición negociadora de la parte asalariada se ve menguada, se requiere un marco reglamentario que establezca la negociación salarial en este nivel. Aún en lo que respecta al trabajo no registrado, en el que las remuneraciones se determinan con prescindencia de la legislación pertinente, los salarios nominales establecidos en el sector formal de la economía constituyen valores de referencia.

En cuanto a los salarios reales, su determinación se ve definida por factores económicos, coyunturales y estructurales, que también pueden reflejar relaciones de poder. Así, si bien todas las corrientes teóricas coinciden en que un incremento de la productividad del trabajo ocasionará, o por lo menos posibilitará un aumento de los salarios reales, para el pensamiento neoclásico los mismos se ven prioritariamente influidos por las fuerzas del mercado, mientras que para la teoría keynesiana guardan relación inversa con el nivel de empleo, de acuerdo a los principios de la productividad marginal del trabajo. En cambio, para el pensamiento marxista resulta central la fase del ciclo en que se encuentre la economía, entendiendo que la remuneración de los asalariados se mueve en forma procíclica, siendo que en las etapas ascendentes del ciclo aumenta la capacidad de negociación de los trabajadores, inclinando la correlación de fuerzas a favor de éstos en el marco de las formas que asume la lucha de clases.

Finalmente, como ya fue visto, todos estos esquemas explicativos deben ser enmarcados en los condicionantes estructurales que define un determinado régimen de acumulación.

\section{Referencias bibliográficas}

Amico, F. (2015). "Los salarios reales en el largo plazo: surgimiento de un nuevo piso estructural de las remuneraciones en argentina". Documento de Trabajo No ${ }^{\circ}$ 67, Marzo de 2015. Centro de Economía y Finanzas para el Desarrollo de la Argentina (CEFID-AR). Buenos Aires.

Astarita, R. (2008). Keynes, poskeynesianos y keynesianos neoclásicos. Apuntes de economía política. 1ra ed. Universidad Nacional de Quilmes. Bernal.

Basualdo, E. (2007). “Concepto de patrón o régimen de acumulación y conformación estructural de la economía". Documento de Trabajo No 1 , Maestría en Economía Política Argentina, Área de Economía y Tecnología de la FLACSO.

Braun O. y Joy L. (1968), “A model of economic stagnation. A case study of the Argentine economy [Un modelo de estancamiento económico. Un caso de estudio de la economía argentina]". En: The Economic Journal, 312

Canitrot, A. (1975). "La experiencia populista de redistribución de ingresos". En: Desarrollo Económico, vol. 15, núm. 59. IDES. Buenos Aires.

Canitrot, A. (1983). "El salario real y la restricción externa de la economía". En: Desarrollo Económico, vol. 23, núm. 91. IDES. Buenos Aires.

Cortés, R. y Marshall, A. (2003). "Salarios, desigualdad y restricción externa bajo distintos regímenes macroeconómicos". En: Realidad Económica, núm. 196. IADE. Buenos Aires.

Fernández, A.L. y González, M. (2019). “El salario y su relación con la restricción externa. Argentina en la posconvertibilidad". En: 14to Congreso Nacional de Estudios del Trabajo. ASET. Agosto de 2019. Buenos Aires.

Friedman, M. (1956). Studies in the Quantity Theory of Money [Estudios de la teoría de la cantidad de dinero]. The University of Chicago Press. Chicago.

Gallo, Marcos Esteban 
Friedman, M. (1960). A Program for Monetary Stability [Programa para la estabilidad monetaria]. Fordham University Press. Nueva York.

Friedman, M. (1969). The Optimun Quantity of Money and Other Essays [Cantidad óptima de dinero y otros ensayos]. Aldine Publishing Company. Chicago.

Friedman, M. y Schwartz, A. (1963). A Monetary History of the United States, 1867-1960 [Historia monetaria de los Estados Unidos, 1867-1960]. Princeton University Press. Princeton.

González, M. (2011). "La relación de largo plazo entre patrones de crecimiento y mercado de trabajo. Un análisis sobre los salarios en Argentina entre 1950 y 2006". Tesis Doctoral. Programa de Doctorado en Ciencias Sociales. FLACSO.

Keynes, J.M. (1939). "Relative Movements of Real Wages and Output [Movimientos relativos de los salarios reales y el producto]". En: Economic Journal, vol. 49, pp. 34-51.

Keynes, J. M. (1974). Teoría general de la ocupación, el interés y el dinero. 2da ed. $8 \mathrm{va}$ reimp. Fondo de Cultura Económica. México.

Kicillof, A. (2004). "El capital según Lord Keynes". En: Revista Nueva Economía. Órgano de la Academia Nacional de Ciencias Económicas de la República de Venezuela. Número doble 21-22.

Kicillof, A. (2007). Fundamentos de la Teoría General. Las consecuencias teóricas de Lord Keynes. 1ra ed. Eudeba. Buenos Aires.

Kicillof, A. (2010). De Smith a Keynes. Siete lecciones de historia del pensamiento económico. Un análisis de los textos originales. 1ra edición. Eudeba. Buenos Aires.

Lucas, R. y Sargent, T. (1979). “After Keynesian
Macroeconomics[Después delamacroeconomía keynesiana]". En: Federal Reserve Bank of Minneapolis, Quarterly Review, vol. 3, núm. 2.

Lucas, R. y Sargent, T.(1981).Rational Expectations and Econometric Practice [Expectativas racionales y práctica econométrica]. University of Minnesota Press. Minneapolis.

Marshall, A. (1890; 1948). Principles of Economics. An introductory volume [Principios de economía. Volumen introductorio]. Macmillan. Londres.

Marx, K. (2004). El capital: crítica de la economía política. Libro primero: el proceso de producción del capital. T. 1. Vol. 1. Siglo XXI Editores Argentina, 1ra ed. 1ra reimp.

Monza, A. (1981). "Una discusión comparada de los distintos enfoques teóricos sobre la determinación del empleo y el salario". Versión preliminar. Curso Regional sobre Empleo, Población y Necesidades Esenciales. Santiago de Chile.

Moore, B. (1988). Horizontalists and Verticalists [Horizontalistas y verticalistas]. Cambridge University Press. Londres.

Neffa, J.C. (2007). "La teoría neoclásica ortodoxa y su interpretación del mercado laboral". En: Teorías económicas sobre el mercado de trabajo. Neoclásicos y nuevos keynesianos. J. C. Neffa (Dir.). CEIL-PIETTE. CONICET. 1ra ed. Fondo de Cultura Económica. Buenos Aires.

Nun, J. (1969). "Superpoblación relativa, ejército industrial de reserva y masa marginal". En: Revista Latinoamericana de Sociología, vol. V, $\mathrm{N}^{\mathrm{o}} 2$, Julio de 1969, Centro de Investigaciones Sociales, Instituto Torcuato Di Tella, Buenos Aires.

Panigo, D. (2006). "Empleo y desempleo en la teoría poskeynesiana". En: Teorías económicas sobre el mercado de trabajo. Marxistas y keynesianos. J. C. Neffa (Dir.). CEIL-PIETTE. CONICET. 
1ra ed. Fondo de Cultura Económica. Buenos Aires.

Patinkin, D. (1959). Dinero, interés y precios. Aguilar. Madrid.

Pérez, P. y Neffa, J.C. (2006). "La teoría general del empleo según J.M. Keynes". En: Teorías económicas sobre el mercado de trabajo. Marxistas y keynesianos. J. C. Neffa (Dir.). CEIL-PIETTE. CONICET. 1ra ed. Fondo de Cultura Económica. Buenos Aires.

Pigou, A. (1933). The Theory of Unemployment [Teoría del desempleo]. Macmillan. Londres.

Sargent, T. y Wallace, N. (1975). ""Rational" Expectations, the Optimal Monetary Instrument, and the Optimal Money Supply Rule [Expectativas "racionales", instrumento monetario ópitmo y regla de la oferta monetaria óptima]". En: The Journal of Political Economy, vol. 83, núm. 2, pp. 241-254.

Serrano, F. (2006). "Pensamiento post-keynesiano y pensamiento marxista". En: Confrontaciones monetarias: marxistas y post-keynesianos en América Latina. A. Girón (coordinadora). 1ra ed. CLACSO. Buenos Aires.

Starosta, G. y Caligaris, G. (2017). Trabajo, valor y capital. De la crítica marxiana de la economía política al capitalismo contemporáneo. 1 ra edición. Universidad Nacional de Quilmes Editorial. Bernal.

Sweezy, P. (1969). Teoría del desarrollo capitalista. 5ta ed. Fondo de Cultura Económica. México. 